\title{
Thermodynamic derivation of the activation energy for ice nucleation
}

\author{
D. Barahona \\ NASA Goddard Space Flight Center, Greenbelt, MD, USA \\ Correspondence to: D. Barahona (donifan.o.barahona@ nasa.gov)
}

Received: 15 May 2015 - Published in Atmos. Chem. Phys. Discuss.: 3 July 2015

Revised: 20 November 2015 - Accepted: 23 November 2015 - Published: 16 December 2015

\begin{abstract}
Cirrus clouds play a key role in the radiative and hydrological balance of the upper troposphere. Their correct representation in atmospheric models requires an understanding of the microscopic processes leading to ice nucleation. A key parameter in the theoretical description of ice nucleation is the activation energy, which controls the flux of water molecules from the bulk of the liquid to the solid during the early stages of ice formation. In most studies it is estimated by direct association with the bulk properties of water, typically viscosity and self-diffusivity. As the environment in the ice-liquid interface may differ from that of the bulk, this approach may introduce bias in calculated nucleation rates. In this work a theoretical model is proposed to describe the transfer of water molecules across the ice-liquid interface. Within this framework the activation energy naturally emerges from the combination of the energy required to break hydrogen bonds in the liquid, i.e., the bulk diffusion process, and the work dissipated from the molecular rearrangement of water molecules within the ice-liquid interface. The new expression is introduced into a generalized form of classical nucleation theory. Even though no nucleation rate measurements are used to fit any of the parameters of the theory the predicted nucleation rate is in good agreement with experimental results, even at temperature as low as $190 \mathrm{~K}$, where it tends to be underestimated by most models. It is shown that the activation energy has a strong dependency on temperature and a weak dependency on water activity. Such dependencies are masked by thermodynamic effects at temperatures typical of homogeneous freezing of cloud droplets; however, they may affect the formation of ice in haze aerosol particles. The new model provides an independent estimation of the activation energy and the homogeneous ice nucleation rate, and it may help to improve the interpretation of experi-
\end{abstract}

mental results and the development of parameterizations for cloud formation.

\section{Introduction}

Ice nucleation in cloud droplets and aerosol particles leads to cloud formation at low temperatures and promotes cloud glaciation and precipitation (Pruppacher and Klett, 1997). In the absence of ice nuclei, it proceeds by homogeneous freezing. Modeling and experimental studies suggest a significant contribution of homogeneous freezing to the formation of clouds in the upper troposphere (Barahona and Nenes, 2011; Barahona et al., 2014; Gettelman et al., 2012; Jensen et al., 2013). The parameterization of ice nucleation is critical to the proper representation of clouds in atmospheric models and is mostly done using empirical formulations (e.g., Lohmann and Kärcher, 2002; Kärcher and Burkhardt, 2008; Barahona et al., 2010, 2014). The most common approach uses the so-called water activity criterion (Koop et al., 2000), where the homogeneous nucleation rate, $J_{\text {hom }}$, is parameterized in terms of the difference between the water activity, $a_{\mathrm{w}}$, and its equilibrium value, $a_{\mathrm{w}, \text { eq. }}$. The greatest advantage of the water activity criterion is that it is independent of the nature of the solute and therefore facilitates the formulation of general parameterizations of ice nucleation (Barahona and Nenes, 2008; Kärcher and Lohmann, 2002; Liu and Penner, 2005).

Empirical formulations provide a simple way to parameterize ice nucleation; however, they provide limited information on the nature of ice formation. Theoretical models help to elucidate the mechanism of ice nucleation and to explain and extend experimental results. Over the last decade 
molecular dynamics (MD) and other detailed methods have provided an unprecedented look at the microscopic mechanism of ice formation (Espinosa et al., 2014). It is known now that the formation of stable ice germs requires the cooperative rearrangement of several molecules (Matsumoto et al., 2002; Moore and Molinero, 2011) and is preceded by structural transformations within the liquid phase (Moore and Molinero, 2011; Bullock and Molinero, 2013). Detailed experiments and MD simulations have shown that instead of forming a single stable structure, several metastable ice structures likely exist during the first stages of ice nucleation (Moroni et al., 2005; Malkin et al., 2012; Russo et al., 2014). There is also a profound relation between anomalies in the properties of water at low temperature and the formation of ice (Buhariwalla et al., 2015), and the relation between low- and highdensity regions within supercooled water and the onset of ice nucleation is starting to be elucidated (Kawasaki and Tanaka, 2010; Singh and Bagchi, 2014; Bullock and Molinero, 2013).

Some theoretical models use mechanistic assumptions to describe the formation of ice. Although less detailed in nature than MD, they are more amenable to the development of parameterizations and to the interpretation of experimental results. The quintessential example of such models is the classical nucleation theory (CNT). According to CNT ice formation proceeds by spontaneous density fluctuations within the liquid phase forming an initial metastable ice germ, which then grows by the incorporation of water molecules from an equilibrium cluster population (Kashchiev, 2000). CNT provides a framework to understand ice nucleation and has been instrumental in the development of parameterizations from experimental data (e.g., Pruppacher and Klett, 1997; Khvorostyanov and Curry, 2009; Murray et al., 2010). On the other hand, $J_{\text {hom }}$ estimated with CNT and using independent estimates of thermodynamic parameters typically results in stark disagreement with measurements (Pruppacher and Klett, 1997; Kawasaki and Tanaka, 2010; Barahona, 2014). Thus, CNT is commonly used semiempirically, fitting several parameters of the theory, most commonly the liquidice interfacial tension, $\sigma_{\mathrm{iw}}$, and the activation energy, $\Delta G_{\text {act }}$, to measured nucleation rates (e.g., Jeffery and Austin, 1997; Khvorostyanov and Curry, 2004; Murray et al., 2010; Ickes et al., 2015).

Using CNT semiempirically has the disadvantage that the theory cannot be decoupled from experimental measurements of $J_{\text {hom }}$. It has been shown that $\sigma_{\text {iw }}$ obtained by fitting CNT to measured nucleation rates tends to be biased high to account for mixing effects neglected in common formulations of CNT (Barahona, 2014). Moreover, the dependency of $\sigma_{\text {iw }}$ on temperature tends to depend on the value of other fitted parameters of the theory (Ickes et al., 2015). Recently, Barahona (2014) (hereinafter B14) introduced a mechanistic model of the ice-liquid interface in terms of thermodynamic variables, without fitting CNT to measured nucleation rates. This was done by hypothesizing the existence of a transition layer around the ice germ, with chemical potential defined by the entropy of the ice and the enthalpy of the liquid, and using the model of Spaepen (1975) to define the interface thickness. This approach was termed the negentropic nucleation framework (NNF). Recent MD simulations showing the existence of a low-density region around the ice germ support the NNF model (Singh and Bagchi, 2014). Introducing NNF into CNT and correcting the nucleation work for mixing effects resulted in good agreement of predicted $J_{\text {hom }}$ with experimental results (Barahona, 2014). NNF was also shown to be consistent with the water activity criterion. On the other hand, even with the inclusion of NNF in CNT, the theory predicts a maximum in $J_{\text {hom }}$ for pure water at around $210 \mathrm{~K}$. Such behavior is at odds with experimental results (Manka et al., 2012) and is ascribed to a strong increase in the activation energy as temperature decreases.

The activation energy controls the flux of water molecules from the bulk of the liquid to the ice germ (Kashchiev, 2000). Most studies estimate $\Delta G_{\text {act }}$ either by direct fit of CNT to measured nucleation rates or from bulk estimates of viscosity, self-diffusivity and dielectric relaxation time (Ickes et al., 2015). The association of bulk properties with $\Delta G_{\text {act }}$ relies on the assumption that the diffusion across the liquid-ice interface is similar to the molecular diffusion in the bulk of the liquid (Kashchiev, 2000). MD results, however, suggest that the properties of water in the vicinity of the ice germ differ from the bulk, casting doubt on such an approach (e.g., Kawasaki and Tanaka, 2010; Singh and Bagchi, 2014). Unlike for the interfacial energy where several theoretical models have been proposed (e.g., Spaepen, 1975; Digilov, 2004; Barahona, 2014), the theoretical treatment of $\Delta G_{\text {act }}$ has been limited. One possible reason is that interface transfer is associated with random fluctuations near the ice-liquid interface and is therefore difficult to treat in terms of macroscopic variables. However, several relations allow us to describe the evolution of fluctuating systems in terms of measurable variables and their relaxation rates. Among them the fluctuation-dissipation theorem that describes the relation between global and local perturbations (Jou et al., 2010) and the fluctuation theorem describing the work distribution in a fluctuating system (Crooks, 1999) have found widespread application in describing the evolution of small systems (Bustamante et al., 2005). With few exceptions (e.g., Røsjorde et al., 2000), such relations have not made their way into descriptions of the ice nucleation process.

In this work a theoretical description of the process leading to the growth of ice germs during ice nucleation is advanced. The proposed model relies on a non-equilibrium view of the interface transfer and leads to the first thermodynamic description of the activation energy for ice nucleation.

\section{Theory}

This section presents the theoretical basis of the proposed model. The ice germ is assumed to form away from the 
Table 1. List of symbols.

\begin{tabular}{|c|c|}
\hline$a_{\mathrm{W}}$ & Activity of water \\
\hline$a_{\mathrm{w}, \mathrm{eq}}$ & Equilibrium $a_{\mathrm{W}}$ between bulk liquid and ice (Koop and Zobrist, 2009) \\
\hline$E, T_{0}$ & Parameters of the VFT equation, 892 and $118 \mathrm{~K}$, respectively (Smith and Kay, 1999) \\
\hline$D$ & Diffusion coefficient for interface transfer, $\mathrm{m}^{2} \mathrm{~s}^{-1}$ \\
\hline$D_{\infty}$ & Self-diffusion coefficient of bulk water, $\mathrm{m}^{2} \mathrm{~s}^{-1}$ \\
\hline$D_{0}$ & Fitting parameter, $3.06 \times 10^{-9} \mathrm{~m}^{2} \mathrm{~s}^{-1}($ Smith and Kay, 1999) \\
\hline$d_{0}$ & Molecular diameter of water, $\mathrm{m}$ \\
\hline$f\left(T, a_{\mathrm{W}}\right)$ & Interface transfer probability \\
\hline$f^{*}$ & Impingement factor, $\mathrm{s}^{-1}$ \\
\hline$G$ & Gibbs free energy, $\mathrm{J}$ \\
\hline$G^{*}$ & Gibbs free energy of the transient state, $\mathrm{J}$ \\
\hline$G_{\text {liq }}, G_{\text {ice }}$ & Gibbs free energy of liquid and ice, respectively, $\mathrm{J}$ \\
\hline$J_{0}, J_{0, \mathrm{CNT}}$ & Preexponential factor calculated from Eqs. (11) and (16), respectively, $\mathrm{m}^{-3} \mathrm{~s}^{-1}$ \\
\hline$J_{\text {hom }}$ & Nucleation rate, $\mathrm{m}^{-3} \mathrm{~s}^{-1}$ \\
\hline$k_{\mathrm{B}}$ & Boltzmann constant, $\mathrm{JK}^{-1}$ \\
\hline$n^{*}$ & Critical germ size \\
\hline$N_{\mathrm{c}}$ & Number of atoms in contact with the ice germ, $5.85 \times 10^{18} \mathrm{~m}^{-2}$ (Pruppacher and Klett, 1997) \\
\hline$n_{\mathrm{t}}$ & Number of formation paths of the transient state, 16 \\
\hline$p_{\mathrm{s}, \mathrm{w}}, p_{\mathrm{s}, \mathrm{i}}$ & Liquid water and ice saturation vapor pressure, respectively, $\mathrm{Pa}$ (Murphy and Koop, 2005) \\
\hline$s$ & Geometric constant of the ice lattice, $1.105 \mathrm{~mol}^{2 / 3}$ (Barahona, 2014) \\
\hline$S_{\mathrm{i}}$ & Saturation ratio with respect to ice \\
\hline$T$ & Temperature, $\mathrm{K}$ \\
\hline$v_{\mathrm{W}}$ & Molecular volume of water in ice (Zobrist et al., 2007), $\mathrm{m}^{-3}$ \\
\hline$W$ & Non-equilibrium work, $\mathrm{J}$ \\
\hline$W_{\text {diss }}$ & Dissipated work, J \\
\hline$Z$ & Zeldovich factor \\
\hline$\Delta a_{\mathrm{W}}$ & $a_{\mathrm{w}}-a_{\mathrm{w}, \mathrm{eq}}$ \\
\hline$\Delta G_{\text {act }}$ & Activation energy for ice nucleation, $\mathrm{J}$ \\
\hline$\Delta G_{\text {hom }}$ & Nucleation work, NNF framework, J \\
\hline$\Delta G_{\mathrm{CNT}}$ & Nucleation work, CNT framework, J \\
\hline$\Delta h_{\mathrm{f}}$ & Heat of fusion of water, $\mathrm{J} \mathrm{mol}^{-1}$, J(Barahona, 2014; Johari et al., 1994)* \\
\hline$\Delta \mu_{\mathrm{f}}$ & Excess free energy of fusion of water, $\mathrm{J}$ \\
\hline$\Gamma_{\mathrm{W}}$ & Molecular surface excess of at the interface, 1.46 (Barahona, 2014; Spaepen, 1975) \\
\hline$\rho_{\mathrm{W}}, \rho_{\mathrm{i}}$ & Bulk density of liquid water and ice, respectively, $\mathrm{Kg} \mathrm{m}^{-3}$ (Pruppacher and Klett, 1997) \\
\hline$\sigma_{\mathrm{iw}}$ & Ice-liquid interfacial energy $\mathrm{J} \mathrm{m}^{-2}$ (Barahona, 2014) \\
\hline$\Omega_{\mathrm{g}}$ & Ice germ surface area $\mathrm{m}^{-2}$ \\
\hline
\end{tabular}

* A change in enthalpy of $50\left(\mathrm{~J} \mathrm{~mol}^{-1}\right)$ was assumed for the transition between cubic and hexagonal ice.

air-liquid interface so that it is not affected by surface tension effects. All the facets of the ice germ are assumed to remain within the bulk of the liquid so that surface-stimulated nucleation (Djikaev, 2008) does not take place. The consequences of this assumption are discussed in Sect. 3. The water molecules incorporated into the ice germ are in close proximity to the ice-liquid interface and diffusion through the bulk of the liquid water can be neglected. This is justified as it is energetically more favorable to incorporate molecules close to the ice germ than those far away from it. Following these considerations the homogeneous nucleation rate can be written in general form as (Kashchiev, 2000)

$J_{\mathrm{hom}}=\frac{Z f^{*}}{v_{\mathrm{w}}} \exp \left(-\frac{\Delta G_{\mathrm{hom}}}{k_{\mathrm{B}} T}\right)$, where $v_{\mathrm{w}}$ is the molecular volume of water in the bulk, $f^{*}$ is the impingement factor of the water molecules for the ice germ, and $Z$ is the Zeldovich factor given by (Kashchiev, 2000)

$Z=\left[\frac{\Delta G_{\mathrm{hom}}}{3 \pi k_{\mathrm{B}} T\left(n^{*}\right)^{2}}\right]^{1 / 2}$,

where $n^{*}$ in the number of water molecules in the ice germ. Other symbols are defined in Table 1. The nucleation work is given by (Barahona, 2014)

$$
\Delta G_{\mathrm{hom}}=\frac{4}{27} \frac{\left[\Gamma_{\mathrm{w}} s\left(\Delta h_{\mathrm{f}}-\Gamma_{\mathrm{w}} k_{\mathrm{B}} T \ln a_{\mathrm{w}}\right)\right]^{3}}{\left[k_{\mathrm{B}} T \ln \left(\frac{a_{\mathrm{w}}^{2}}{a_{\mathrm{w}, \text { eq }}}\right)\right]^{2}},
$$


where $a_{\mathrm{w}}$ is the water activity in the liquid and $a_{\mathrm{w}, \text { eq }}$ is the value of $a_{\mathrm{w}}$ at thermodynamic equilibrium with ice at the same temperature. $\Gamma_{\mathrm{w}}=1.46$ is the coverage of the iceliquid interface, and $s=1.105$ defines the lattice geometry of the ice germ. The value of $\Gamma_{\mathrm{w}}$ results from the explicit construction of the interface following these rules: (i) maximize the density; (ii) disallow octahedral holes; and (iii) give preference to tetrahedral holes (Spaepen, 1975). The value of $s$ is obtained assuming that the germ has a staggered structure lying somewhere between cubic and hexagonal ice (Malkin et al., 2012). Compared to common expressions for $\Delta G_{\text {hom }}$ derived from CNT, Eq. (3) has the advantage that it does not depend on an explicit parameterization of $\sigma_{\mathrm{iw}}$, for which there is large uncertainty. The application of Eqs. (1) to (3) has been shown to reproduce observed freezing temperatures (Barahona, 2014).

The impingement factor is the frequency of attachment of water molecules to the ice germ. For steady-state nucleation it is given by (Kashchiev, 2000)

$f^{*}=\frac{\gamma D Z_{1} \Omega}{d_{0}}$,

where $\gamma \approx 1$ is the sticking coefficient, $D$ the diffusion coefficient for interface transfer, $\Omega$ the surface area of the germ, $d_{0}$ the molecular diameter and $Z_{1} \approx v_{\mathrm{w}}^{-1}$ the monomer concentration. In this work $D$ is understood as an "effective diffusivity" since it parameterizes processes that are not necessarily diffusive in nature, like molecular rearrangement and reorientation, but that result in the incorporation of water molecules into the ice germ.

Uncertainty in the determination of $f^{*}$ results mostly from the calculation of $D$, which may differ from the bulk selfdiffusivity of water. The most commonly used approximation to $D$ was derived from transition state theory by Turnbull and Fisher (1949) (see Sect. 2.3), who assumed that the activation energy for interface transfer is similar to that of the bulk liquid; however, the vibration frequency is that of an elemental reaction in the gas phase. This approximation tends to underpredict the preexponential factor in Eq. (1) at low temperature (Ickes et al., 2015). Here an alternative expression is proposed assuming that $D$ can be expressed in the form

$D=f\left(T, a_{\mathrm{w}}\right) D_{\infty}$,

where $D_{\infty}$ is self-diffusivity of water in the bulk. The function $f\left(T, a_{\mathrm{w}}\right)$ is introduced to distinguish between the bulk diffusion coefficient $D_{\infty}$ and the diffusion coefficient across the interface, $D$. Since $D_{\infty}$ has been measured to $T \sim 180 \mathrm{~K}$ (Smith and Kay, 1999), $D$ can be calculated provided that a suitable form $f\left(T, a_{\mathrm{w}}\right)$ is known. In principle $f\left(T, a_{\mathrm{w}}\right)$ can be found by fitting nucleation rate measurements. It is, however, desirable to obtain an expression for $f\left(T, a_{\mathrm{w}}\right)$ independent of $J_{\text {hom }}$. To this end a heuristic approach is developed as follows.

\subsection{Activation energy}

Similarly to Turnbull and Fisher (1949) it is assumed that interface transfer requires the formation of a transient state. However, instead of each molecule moving independently across the interface, the formation of the transient state requires the collective rearrangement of several water molecules. The probability of such a collective arrangement is given by $f\left(T, a_{\mathrm{w}}\right)$. This view does not imply that water is incorporated in clusters into the ice but rather that the rearrangement of the molecules facilitates the incorporation of each molecule into the preexisting ice lattice. Such a lattice is assumed to be the exposing surface of a metastable ice germ. In other words, the breaking of hydrogen bonds is a necessary but not sufficient condition for the incorporation of water molecules into the ice germ. Molecular rearrangement is required for interface transfer and requires surpassing an energy barrier. This view is supported by MD simulations showing the increase in the fraction of four-coordinated water prior to nucleation (e.g., Moore and Molinero, 2011; Matsumoto et al., 2002) and theoretical models where the selfdiffusion of supercooled liquids is controlled by their configurational entropy (Adam and Gibbs, 1965).

A key aspect of the transient state is that it has a higher free energy than that of bulk water, which stems from the lowering of the entropy as molecules organize into an icelike structure and an increase in the enthalpy from the breaking of hydrogen bonds (e.g., Bullock and Molinero, 2013). Macroscopically, the formation of a transient state is thermodynamically unfavorable; however, microscopically it can be reached by spontaneous fluctuation. The probability of a molecule fluctuating to a given state is associated with the non-equilibrium work required to reach such a state. Interface transfer requires the spontaneous organization of water molecules into ice-like structures with the probability described by $f\left(T, a_{\mathrm{w}}\right)$. Thus, if $W$ is the work required for collective rearrangement, $f\left(T, a_{\mathrm{w}}\right)$ must be equal to the work probability, $P(W)$. Molecules near the interface can fluctuate in any direction either by doing work on the system or vice versa, by the system doing work on them. The work distribution in such systems is governed by the fluctuation theorem (FT) (Crooks, 1999):

$\frac{P(W)}{P(-W)}=\exp \left(\frac{W-\Delta G}{k_{\mathrm{B}} T}\right)$,

where $P(W)$ and $P(-W)$ correspond to the work probability of the forward and reversed process between two states of a system, respectively; $\Delta G$ is their equilibrium free energy difference, and $W$ is the non-equilibrium work between the two states. Considering only those subsystems that move across the interface, we assume $P(W)+P(-W)=1$. Using this and setting $f\left(T, a_{\mathrm{w}}\right)=P(W)$, Eq. (6) can be rearranged into

$f\left(T, a_{\mathrm{w}}\right)=\left[1+\exp \left(-\frac{W-\Delta G}{k_{\mathrm{B}} T}\right)\right]^{-1}$. 


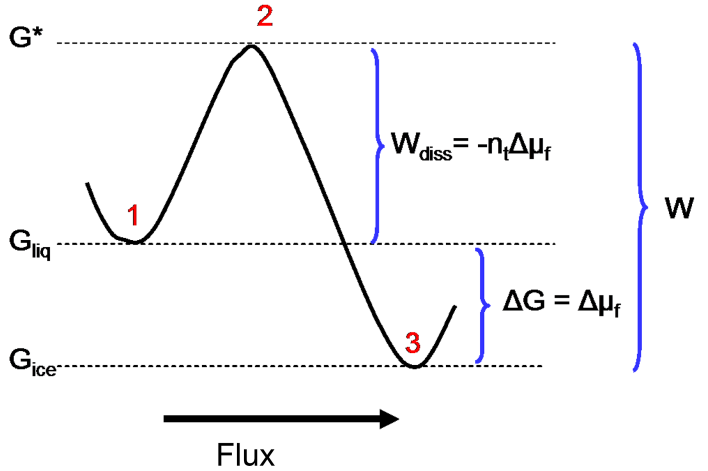

Figure 1. Simplified energy landscape of water molecules in the ice-liquid interface during interface transfer. States 1 and 3 correspond to metastable equilibrium positions in the liquid and ice, respectively, and 2 corresponds to the transient state. $G_{\text {ice }}, G_{\text {liq }}$ and $G^{*}$ correspond to the Gibbs free energy of ice, liquid and the transient state, respectively. $W$ and $W_{\text {diss }}$ are the total non-equilibrium work and the dissipated work, respectively. $\Delta \mu_{\mathrm{f}}$ is the excess free energy of water and $n_{t}$ is defined in Sect. 2. The bold arrow represents the direction of the net flux of water molecules during the growth of the ice germ.

Equation (6) is one of the few thermodynamic relations valid in systems away from equilibrium (Bustamante et al., 2005). In writing Eq. (6) it has been assumed that the system is incompressible so that $\Delta G$ approximates the Helmholtz free energy difference. The difference $W-\Delta G$ approximates the dissipated work, $W_{\text {diss }}$, between the two states (Bustamante et al., 2005; Jou et al., 2010). Equation (6) relates the macroscopic response of a thermodynamic system to its microscopic dynamics under the assumption that the latter is stochastic and Markovian. These are typically valid assumptions at conditions away from the glass transition temperature (Crooks, 1999). A further assumption is that of microscopic reversibility, which requires that thermodynamic potentials can be locally defined within the liquid. This is guaranteed near equilibrium; however, in non-equilibrium transformations it only holds for systems starting at local equilibrium. Thus, the application of FT for interface transfer is valid only if each molecule can be considered in equilibrium with its local environment within the liquid (Crooks, 1999). Again, this is satisfied at $T$ higher than the glass transition temperature, which is the case for most systems of atmospheric interest.

Consider the simplified energy landscape of the water molecules within the interface shown in Fig. 1. The states 1 and 3 refer to pseudoequilibrium positions (i.e., metastable states) on the liquid and the ice side, respectively, and 2 corresponds to the transient state. We define the path $1 \rightarrow 3$ as the forward and $3 \rightarrow 1$ as the reversed process. At thermodynamic equilibrium, the forward and reversed processes are equally probable, there is no work dissipation, and the molecules move freely across the interface without the formation of a transient state. For this situation $\Delta G=G_{\text {liq }}-$
$G_{\text {ice }}=W=0$, with $G_{\text {liq }}$ and $G_{\text {ice }}$ being the Gibbs free energy of bulk liquid and ice, respectively. For the ice germ to grow, the net flux of molecules should correspond to the direction of the forward process, the path $1 \rightarrow 3$.

As water supercools it moves away from equilibrium and an energy barrier for interface transfer is created, i.e., $W>0$ and $\Delta G<0$. To estimate the dissipated work in this situation it is easier to consider the reversed process (path $3 \rightarrow 1$, Fig. 1). This corresponds to molecules spontaneously leaving the ice lattice and moving into the bulk of the liquid. To move away from the ice lattice, molecules should pass through the transient state (path $3 \rightarrow 2$, Fig. 1) and gain energy equal to $G^{*}-G_{\text {ice }}, G^{*}$ being the energy of the transient state, which is later dissipated into the bulk of the liquid. On the other hand, if such a process could be carried out in a completely reversible manner, then a transient state would not form and molecules would only need $G_{\text {liq }}-G_{\text {ice }}$ to leave the lattice (i.e., a hypothetical path $3 \rightarrow 1$ without passing by 2 ; not shown). Thus, for the reversed process, $W-\Delta G=G^{*}-G_{\text {liq }}$. If the subsystem follows the same trajectory but in the opposite direction, i.e., the forward process (path $1 \rightarrow 3$; Fig. 1), then $W-\Delta G=-\left(G^{*}-G_{\text {liq }}\right)$. That is, the energy dissipated when water molecules are incorporated into the ice germ is equal to their activation energy, i.e., interface transfer is a dissipative process. Using this in Eq. (7), we obtain

$$
f\left(T, a_{\mathrm{w}}\right)=\left[1+\exp \left(\frac{G^{*}-G_{\text {liq }}}{k_{\mathrm{B}} T}\right)\right]^{-1} .
$$

A molecule following the forward process (path $1 \rightarrow 3$, Fig. 1) experiences a net energy change equal to $G_{\text {ice }}-G_{\text {liq }}=$ $\Delta \mu_{\mathrm{f}}, \Delta \mu_{\mathrm{f}}$ being the excess free energy of fusion of water. This is equal to the energy change in a molecule moving between two pseudoequilibrium positions. Thus, dissipation should result mostly from collective rearrangement of several molecules, i.e., from the formation of the transient state. This is consistent with the assumption of internal reversibility used in Eq. (6). Moreover, it suggests that the difference $G^{*}-G_{\text {liq }}$ should be proportional to $\Delta \mu_{\mathrm{f}}$ since $\Delta \mu_{\mathrm{f}}$ is the minimum energy change experienced by each molecule during interface transfer.

To find the proportionality constant between $G^{*}-G_{\text {liq }}$ and $\Delta \mu_{\mathrm{f}}$, we consider the entropy change associated with the formation of the transient state. In the ice lattice, water molecules have minimal configurational entropy and can be assumed to vibrate around fixed positions. When moving towards the liquid, they experience an increase in entropy that can be written in the form $\Delta S_{\mathrm{f}}=k_{\mathrm{B}} \log \omega, \omega$ being the number of possible configurations of the liquid, and $\Delta S_{\mathrm{f}}$ the excess entropy of fusion. If there are $n_{\mathrm{t}}$ "trajectories" in which the transient state can be formed, then the number of possible configurations at the transient state would be $\omega^{n_{\mathrm{t}}}$. Thus, the change in entropy between a pseudoequilibrium position in the ice and the transient state can be written as $k_{\mathrm{B}} \log \omega^{n_{\mathrm{t}}}=n_{\mathrm{t}} \Delta S_{\mathrm{f}}$. By extension, the change in entropy between the transient state and a pseudoequilibrium 
position in the liquid would be $-n_{\mathrm{t}} \Delta S_{\mathrm{f}}$. Since work dissipation is proportional to the generation of entropy (Bustamante et al., 2005), the work required for interface transfer must be determined by entropy changes. Thus, we make $G^{*}-G_{\text {liq }} \approx-n_{\mathrm{t}} \Delta \mu_{\mathrm{f}}, n_{\mathrm{t}}$ being the number of possible paths in the formation of the transient state. Inserting this into Eq. (8) we obtain

$f\left(T, a_{\mathrm{w}}\right)=\left[1+\exp \left(-\frac{n_{\mathrm{t}} \Delta \mu_{\mathrm{f}}}{k_{\mathrm{B}} T}\right)\right]^{-1}$.

Using $\Delta \mu_{\mathrm{f}}=-k_{\mathrm{B}} T \ln \left(\frac{a_{\mathrm{w}}}{a_{\mathrm{w}, \mathrm{eq}}}\right)$, Eq. (9) can be written in terms of $a_{\mathrm{w}}$ :

$f\left(T, a_{\mathrm{w}}\right)=\left[1+\left(\frac{a_{\mathrm{w}}}{a_{\mathrm{w}, \mathrm{eq}}}\right)^{n_{\mathrm{t}}}\right]^{-1}$,

where $a_{\mathrm{w}, \mathrm{eq}}$ is the equilibrium water activity.

To complete the derivation of $f\left(T, a_{\mathrm{w}}\right)$ it is necessary to specify $n_{\mathrm{t}}$. MD simulations show that the onset of nucleation is accompanied by an increase in the number of fourcoordinated molecules (Moore and Molinero, 2011; Matsumoto et al., 2002). In the view proposed here this means that for each molecule that is incorporated into the ice germ at least four neighboring molecules would rearrange themselves into the transient state. A geometrical argument is used to count the possible number of trajectories for this process. Essentially, it is assumed that in the transient state, the water molecules exhibit coordination numbers between 0 and 4 . Thus, each molecule can be coordinated with zero, one, two, three or four other molecules, each combination corresponding to a different trajectory of the system. The total number of trajectories is then given by $n_{\mathrm{t}}=2^{4}=16$.

\subsection{Nucleation rate}

Collecting Eqs. (2) to (5) into Eq. (1) we obtain,

$$
\begin{aligned}
J_{\text {hom }} & =\left(\frac{Z \Omega}{v_{\mathrm{w}}}\right) \frac{D_{\infty} f\left(T, a_{\mathrm{w}}\right)}{v_{\mathrm{w}} d_{0}} \exp \left(-\frac{\Delta G_{\mathrm{hom}}}{k_{\mathrm{B}} T}\right) \\
& =J_{0} \exp \left(-\frac{\Delta G_{\mathrm{hom}}}{k_{\mathrm{B}} T}\right),
\end{aligned}
$$

where $J_{0}$ is referred to as the preexponential factor. Since water is a glass-forming substance, the temperature dependency of $D_{\infty}$ can be described by the Vogel-Fulcher-Tammann (VFT) equation:

$D_{\infty}=D_{0} \exp \left[-\frac{E}{\left(T-T_{0}\right)}\right]$,

where $D_{0}, E$ and $T_{0}$ are fitting parameters (Table 1, Smith and Kay (1999)). At temperatures relevant for homogeneous ice nucleation, the exponential term in Eq. (8) is expected to be much greater than 1 (although this is not the case when $\left.a_{\mathrm{w}} \sim a_{\mathrm{w}, \mathrm{eq}}\right)$. Using this and inserting Eqs. (10) and (12) into Eq.(11), we obtain

$$
\begin{aligned}
J_{\text {hom }} & \approx\left(\frac{D_{0}}{v_{\mathrm{w}} d_{0}}\right)\left(\frac{Z \Omega}{v_{\mathrm{w}}}\right) \exp \left\{-\frac{1}{k_{\mathrm{B}} T}\left[\frac{k_{\mathrm{B}} T E}{\left(T-T_{0}\right)}\right.\right. \\
& \left.\left.+k_{\mathrm{B}} T n_{\mathrm{t}} \ln \left(\frac{a_{\mathrm{w}}}{a_{\mathrm{w}, \mathrm{eq}}}\right)+\Delta G_{\mathrm{hom}}\right]\right\} .
\end{aligned}
$$

Equation (13) has the form proposed by Turnbull and Fisher (1949). Thus, the activation energy can be derived as

$\Delta G_{\mathrm{act}}=k_{\mathrm{B}} T\left[\frac{E}{\left(T-T_{0}\right)}+n_{\mathrm{t}} \ln \left(\frac{a_{\mathrm{w}}}{a_{\mathrm{w}, \mathrm{eq}}}\right)\right]$.

Equation (14) shows two contributions to the energy barrier for water transfer to the ice germ. The first term on the right hand side of Eq. (14) results from the breaking of hydrogen bonds in the liquid phase, i.e., the bulk diffusion process. The second term represents an additional energy barrier resulting from the entropy cost of molecular rearrangement within the ice-liquid interface. Substituting Eq. (14) into Eq. (13) we finally obtain

$$
J_{\text {hom }} \approx\left(\frac{D_{0}}{v_{\mathrm{w}} d_{0}}\right)\left(\frac{Z \Omega}{v_{\mathrm{w}}}\right) \exp \left(-\frac{\Delta G_{\mathrm{act}}+\Delta G_{\mathrm{hom}}}{k_{\mathrm{B}} T}\right) .
$$

\subsection{Common form of CNT}

In most studies CNT is used in a more simplified form than presented in Eq. (1) (e.g., Khvorostyanov and Curry, 2004; Zobrist et al., 2007; Murray et al., 2010; Ickes et al., 2015). Typically, the expression of Einstein (1956) is used to relate diffusivity and viscosity and the activation energy of water is assumed to have the same value as in the bulk (Kashchiev, 2000). Other assumptions include a semi-spherical ice germ and negligible mixing effects during the germ formation (Barahona, 2014). These considerations lead to the commonly used CNT expression for $J_{\text {hom }}$ (Turnbull and Fisher, 1949):

$$
\begin{aligned}
J_{\mathrm{hom}} & =\left(\frac{N_{\mathrm{c}} k_{\mathrm{B}} T}{h} \frac{\rho_{\mathrm{w}}}{\rho_{\mathrm{i}}}\right)\left(\frac{Z \Omega}{v_{\mathrm{w}}}\right) \exp \left(-\frac{\Delta G_{\mathrm{act}}+\Delta G_{\mathrm{CNT}}}{k_{\mathrm{B}} T}\right) \\
& =J_{0, \mathrm{CNT}} \exp \left(-\frac{\Delta G_{\mathrm{CNT}}}{k_{\mathrm{B}} T}\right)
\end{aligned}
$$

where $N_{\mathrm{c}}$ is the number of atoms in contact with the ice germ and $\rho_{\mathrm{w}}$ and $\rho_{\mathrm{i}}$ are the bulk liquid water and ice density, respectively. $\Delta G_{\mathrm{CNT}}$ is the energy of formation of the ice germ, commonly written in the form (Pruppacher and Klett, 1997)

$\Delta G_{\mathrm{CNT}}=\frac{16 \pi \sigma_{\mathrm{iw}}^{3} v_{\mathrm{w}}^{2}}{3\left(k_{\mathrm{B}} T \ln S_{\mathrm{i}}\right)^{2}}$,

where $\sigma_{\mathrm{iw}}$ is the ice-liquid interfacial energy and $S_{\mathrm{i}}$ is the saturation ratio with respect to ice. Other symbols are defined in Table 1. When using Eqs. (16) and (17), $\Delta G_{\text {act }}$ and $\sigma_{\text {iw }}$ are typically considered free parameters. 

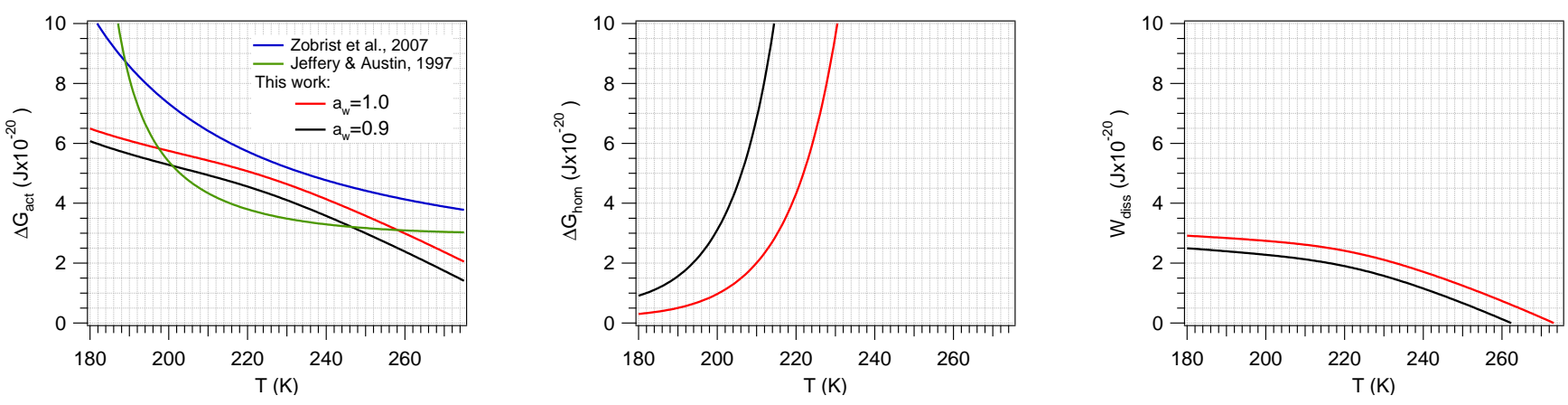

Figure 2. Activation energy (left panel), nucleation work (middle panel) and dissipated work (right panel). Black and red lines correspond to $a_{\mathrm{w}}=0.9$ and $a_{\mathrm{w}}=1$, respectively.

\section{Discussion}

As temperature decreases the configurational entropy of water decreases, increasing the energy required to break hydrogen bonds; thus, the self-diffusivity of water decreases (Adam and Gibbs, 1965). Similarly, as $T$ decreases the energy associated with the molecular rearrangement within the interface increases, which results from a more negative excess energy of fusion. The latter can also be understood as an increase in the irreversibility of the liquid-ice transformation as the system moves away form thermodynamic equilibrium, therefore increasing the dissipated work, $W_{\text {diss. }}$. As a result, $\Delta G_{\text {act }}$ increases monotonically as $T$ decreases (Fig. 2). By definition, the rearrangement component of $\Delta G_{\text {act }}, W_{\text {diss }}$, for $a_{\mathrm{w}}=1$ is equal to 0 at $T=273.15 \mathrm{~K}$, i.e., the equilibrium temperature of the bulk ice-liquid system. For $T<250 \mathrm{~K}$, it corresponds to about half of $\Delta G_{\text {act }}$.

An important aspect of Eq. (14) is that it predicts an effect of water activity on the activation energy. The dependency of $\Delta G_{\text {act }}$ on $a_{\mathrm{w}}$ is, however, much weaker than on $T$. Decreasing $a_{\mathrm{w}}$ from 1.0 to 0.9 leads to a decrease of only about $10 \%$ decrease $\Delta G_{\text {act }}$ (Fig. 2). This is caused by a lowering in the dissipated work, $W_{\text {diss }}=-n_{\mathrm{t}} \Delta \mu_{\mathrm{f}}$, with decreasing $a_{\mathrm{w}}$. Lowering $a_{\mathrm{w}}$ reduces the chemical potential of water but not that of ice as it is likely that no solute is incorporated into the ice germ during the early stages of ice formation (Barahona, 2014), therefore reducing $\Delta \mu_{\mathrm{f}} . \Delta G_{\text {hom }}$ (Eq. 3 ) is much more sensitive to $a_{\mathrm{w}}$ and dominates the dependency of $J_{\text {hom }}$ on $a_{\mathrm{w}}$.

Empirical estimates of $\Delta G_{\text {act }}$ have been developed in several studies and were recently reviewed by Ickes et al. (2015). The authors found that the usage of the expression derived by Zobrist et al. (2007) from self-diffusivity measurements (Smith and Kay, 1999), along with the fit of Reinhardt and Doye (2013) for $\sigma_{\text {iw }}$, into Eq. (16) produced the best comparison with experimental results. Here the empirical expression for $\sigma_{\text {iw }}$ proposed in B14, derived from fitting Eq. (1) to the model of Koop et al. (2000), is used as it is the only available correlation that includes an explicit dependency of $\sigma_{\text {iw }}$ on $a_{\mathrm{w}}$. Usage of the B14 correlation also ensures that $\Delta G_{\mathrm{CNT}} \approx \Delta G_{\mathrm{hom}}$ since it empirically accounts for mixing effects. The Zobrist et al. (2007) correlation results from taking the derivative of the exponential argument of Eq. (12) in the form

$\Delta G_{\mathrm{act}, \mathrm{Z} 07}=\frac{k_{\mathrm{B}} T^{2} E}{\left(T-T_{0}\right)^{2}}$.

Equation (18) gives $\Delta G_{\text {act }}$ around the mean of common models used in the literature (see Fig. 1 of Ickes et al., 2015). Thus, the model of Zobrist et al. (2007) will be used as a benchmark for comparison. However, $\Delta G_{\text {act }}$ calculated using the correlation of Jeffery and Austin (1997) is also presented in Fig. 2 for reference. Although the latter is also derived from the bulk properties of water, it typically results in values of $\Delta G_{\text {act }}$ lower than $\Delta G_{\text {act,Z07. }}$.

Because the contribution of the breaking of hydrogen bonds to $\Delta G_{\text {act }}$ is taken into account in both models, Eqs. (14) and (18) show similar dependencies on $E$ and $T_{0}$, since they define $D_{\infty}$. However, the two expressions are fundamentally different. In the model of Zobrist et al. (2007) the relation between $D_{\infty}$ and $\Delta G_{\text {act }}$ is hypothesized a priori, while in this work it results from an explicit consideration of the thermodynamics of interface transfer. Moreover, the contribution to $\Delta G_{\text {act }}$ from molecular rearrangement, neglected in Zobrist et al. (2007), is always positive for nucleation (i.e., Eq. 14 is never evaluated at $a_{\mathrm{w}}=a_{\mathrm{w} \text {, eq }}$ since it implies equilibrium conditions for which $J_{\text {hom }}=0$ ).

Figure 2 shows that $\Delta G_{\text {act,Z07 increases almost quadrati- }}$ cally as $T$ decreases. The expression of Jeffery and Austin (1997) results in an even stronger increase in $\Delta G_{\text {act }}$ for $T<$ $200 \mathrm{~K}$. This feature is common in empirical models (Ickes et al., 2015). In general $\Delta G_{\text {act,Z07 }}$ is larger than $\Delta G_{\text {act }}$ calculated from Eq. (14). The latter increases almost linearly as $T$ decreases instead of the quadratic increase of $\Delta G_{\text {act,Z07. }}$ $\Delta G_{\text {act,Z07 }}$ and Eq. (14) are the closest around $T \approx 235 \mathrm{~K}$, which is near the nominal homogeneous freezing threshold of water droplets. The difference between the two grows larger with decreasing temperature since the work dissipated in the formation of the transient state increases (Sect. 2.1); at $T=180 \mathrm{~K}, \Delta G_{\text {act,Z07 }}$ is higher than $\Delta G_{\text {act }}$ by almost a factor of 2 . 


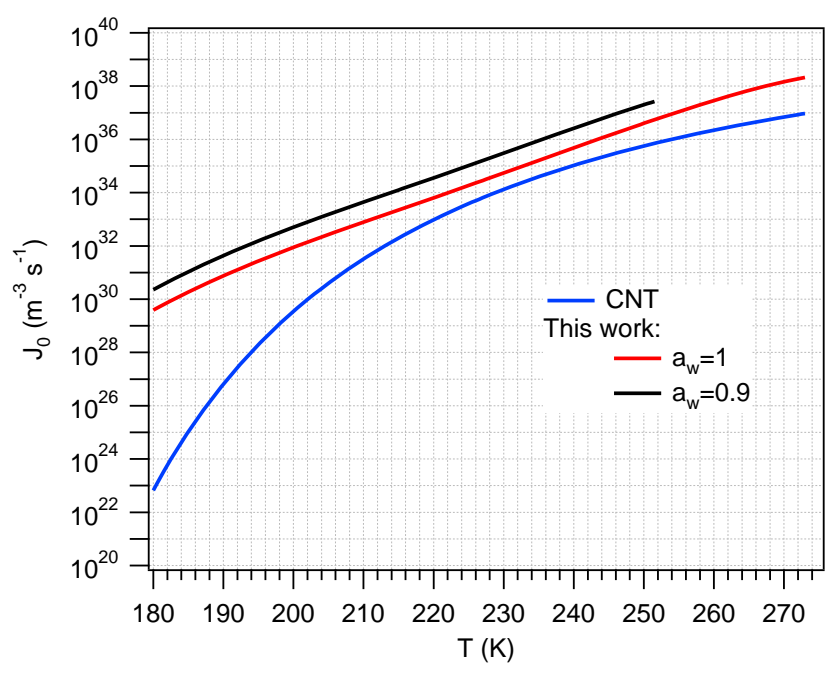

Figure 3. Preexponential factor using the common form of CNT (Sect. 2.3) and the model presented in this work, Eq. (11). For CNT the formulation of Zobrist et al. (2007) was used to calculate $\Delta G_{\text {act }}$.

Figure 3 compares the preexponential factor calculated from Eq. (11) to the common CNT formulation, Eq. (16). Equation (18) was used to calculate $\Delta G_{\text {act }}$ in the latter. For $T<240 \mathrm{~K}$, the factors $\left(\frac{D_{0}}{v_{\mathrm{w}} d_{0}}\right)$ and $\left(\frac{N_{\mathrm{c}} k_{\mathrm{B}} T}{h} \frac{\rho_{\mathrm{W}}}{\rho_{\mathrm{i}}}\right)$ differ by less than a factor of 2 . Thus, the difference between $J_{0}$ and $J_{0, \mathrm{CNT}}$ is almost entirely due to $\Delta G_{\text {act }}$. For $T>230 \mathrm{~K}$, the use of either $\Delta G_{\text {act, } 07}$ or Eq. (14) introduces a difference of less than 2 orders of magnitude into $J_{0}$. However, for $T<230 \mathrm{~K}$ using $\Delta G_{\text {act, } 07}$ leads to a much faster decrease in $J_{0}$ than with Eq. (14), which is explained by the quadratic increase in $\Delta G_{\text {act }, 07}$ as $T$ decreases. At $180 \mathrm{~K}$, they differ by almost 10 orders of magnitude. As expected, lowering the water activity slightly increases $J_{0}$ since $\Delta G_{\text {act }}$ is slightly reduced.

Despite the noticeable dependency of $\Delta G_{\text {act }}$ on $T, J_{\text {hom }}$ is mostly sensitive to variation in $\Delta G_{\text {act }}$ at low $T$. This is illustrated in Fig. 2. For $a_{\mathrm{w}}=1$ and $T>230 \mathrm{~K}, \Delta G_{\mathrm{hom}} \gg$ $\Delta G_{\text {act }}$, i.e., the nucleation rate is completely controlled by the nucleation work. As $T$ decreases, $\Delta G_{\text {hom }}$ and $\Delta G_{\text {act }}$ become comparable, and for $T<200 \mathrm{~K}, J_{\text {hom }}$ is mainly controlled by $\Delta G_{\text {act }}$. Since most experimental measurements of $J_{\text {hom }}$ are carried out around $235 \mathrm{~K}$, the lack of sensitivity of $J_{\text {hom }}$ to $\Delta G_{\text {act }}$ at these conditions may lead to the incorrect notion that $\Delta G_{\text {act }}$ is constant. Such a misconception may not be critical for the homogeneous freezing of pure water at atmospheric conditions since it rarely occurs at $T<230 \mathrm{~K}$. However, it may introduce error in $J_{\text {hom }}$ for $a_{\mathrm{w}}<1$ (Fig. 2, black lines) since $\Delta G_{\text {hom }}$ and $\Delta G_{\text {act }}$ become comparable at temperatures relevant to the formation of cirrus from haze aerosol particles (Barahona and Nenes, 2008).

As direct measurements of $\Delta G_{\text {act }}$ are not available, the skill of $\Delta G_{\text {act }}$ in reproducing experimental measurements is assessed through an evaluation of $J_{\mathrm{hom}}$. For common formulations of CNT (Sect. 2.3), this has the caveat that such a comparison is influenced by the specification of other parameters of the theory. This is not the case when using the NNF formulation (Eq. 3) since it does not explicitly depend on $\sigma_{\mathrm{iw}}$. It was shown in B14 that using $\Delta G_{\mathrm{act}, 07}$ and Eq. (3) in Eq. (16) reproduced measured $J_{\text {hom }}$ for $T>230 \mathrm{~K}$. The results of B14 for $a_{\mathrm{w}}=1$ are shown in Fig. 4 along with several experimental measurements, empirical correlations, and results from the formulation of CNT presented in Sect. 2.3.

Most of the measurements of $J_{\text {hom }}$ are carried out using small droplets with a relatively high surface-to-volume ratio. Surface-stimulated nucleation (SSN) thus may take place leading to values of $J_{\text {hom }}$ higher than the volume-based theory presented here (Djikaev, 2008). However, the experimental evidence for the predominance of SSN is still conflicting (Sigurbjörnsson and Signorell, 2008; Kay et al., 2003). SSN requires an ice germ growing with a particular orientation so that at least one of its "facets" is aligned with the droplet-vapor interface. However, it is not clear that the exposed facet would have the same interfacial tension as bulk ice (Kay et al., 2003). Moreover, SSN is a thermodynamic effect, i.e., it decreases $\Delta G_{\text {hom }}$ compared to volume-based nucleation. As shown in Fig. 2, at a low temperature, $J_{\text {hom }}$ is increasingly less controlled by $\Delta G_{\mathrm{hom}}$ and more dependent on $\Delta G_{\text {act }}$, which would limit the effect of SSN on $J_{\text {hom }}$. Therefore we limit the comparison in Figs. 4 and 5 to those data sets where $J_{\text {hom }}$ is interpreted as dominated by volumebased ice nucleation.

Compared to the formulation of B14, $J_{\text {hom }}$ from Eq. (11) only differs in the specification of $J_{0}$, which mainly depends on $\Delta G_{\text {act }}$. As expected, for $T>230$ and $a_{\mathrm{w}}=1$ the formulation of B14 and Eq. (11) produces similar $J_{\text {hom }}$ and within experimental variability and model uncertainty (typically about 3 orders of magnitude) of measured values. Notably $J_{\text {hom }}$ predicted by NNF is very close to the data of Riechers et al. (2013), who used a microfluidic device to obtain an accurate estimation of $T$. For $T<230 \mathrm{~K}, J_{\text {hom }}$ from B14 is much lower than measured values (by up to 9 orders of magnitude), which is also the case for the CNT formulation, Eq. (16), when using $\Delta G_{\text {act, } 07}$. In both formulations $J_{\text {hom }}$ decreases for $T$ below $210 \mathrm{~K}$, which results from an strong increase in $\Delta G_{\text {act, } 07}$ and a decrease in $J_{0}$. Using the fit of Reinhardt and Doye (2013) for $\sigma_{\text {iw }}$ and $\Delta G_{\text {act, } 07}$ also leads to underestimation of $J_{\text {hom }}$ at low $T$, consistent with the results of Ickes et al. (2015). At the same conditions, Eq. (11) predicts a higher $J_{\text {hom }}$ within experimental uncertainty of measurements. Using Eq. (14) within the CNT formulation, Eq. (16), leads to a similar result. Thus, the higher $J_{\text {hom }}$ and the better agreement with the experimental measurements at low $T$ results from the usage of the formulation of $\Delta G_{\text {act }}$ presented here.

Most experimental measurements of $J_{\text {hom }}$ have been carried out for $a_{\mathrm{w}}=1$. However, homogeneous freezing for $a_{\mathrm{w}}<1$ is important for the formation of cirrus at low $T$ (e.g., Koop et al., 2000). Figure 5 shows $J_{\text {hom }}$ for $a_{\mathrm{w}}=0.9$ from Eqs. (16) and (11), using $\Delta G_{\text {act, } 07}$ and Eq. (14) to compute 


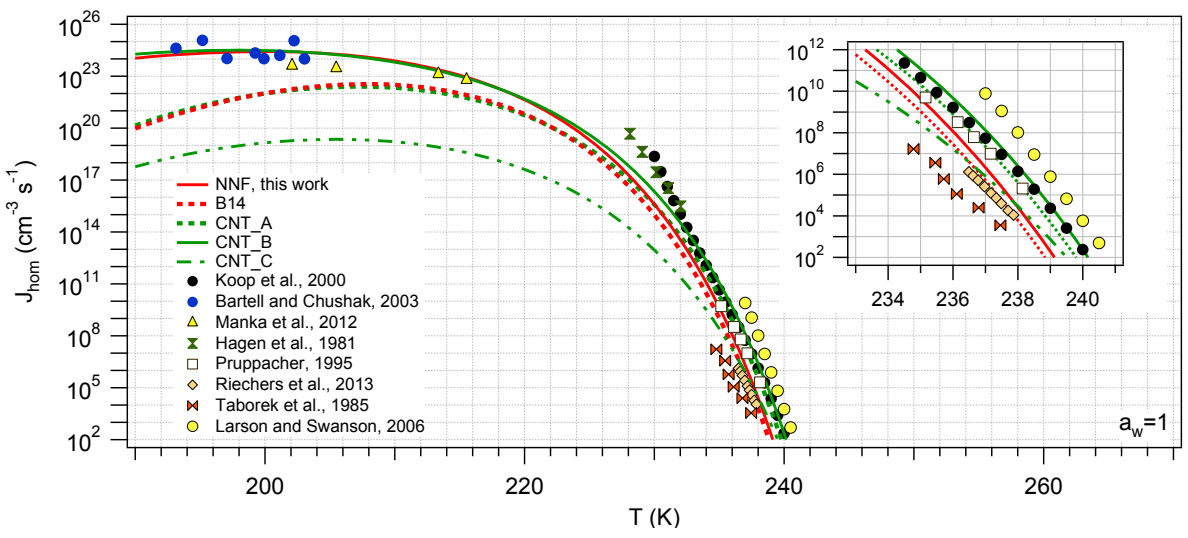

Figure 4. Homogeneous ice nucleation rate calculated using Eq. (16) (labeled "CNT") and Eq. (11) (labeled "NNF"). For CNT_A $\Delta G_{\text {act }}$ was defined as in Zobrist et al. (2007), and for CNT_B it was calculated using Eq. (14); in both cases the empirical expression for $\sigma_{\text {iw }}$ from Barahona (2014) was used. For CNT_C the expression of Zobrist et al. (2007) was used for $\Delta G_{\text {act }}$ and $\sigma_{\text {iw }}$ was calculated using the fit of Reinhardt and Doye (2013). For the model of Koop et al. (2000), $a_{\mathrm{w}, \mathrm{eq}}$ was defined as in Koop and Zobrist (2009). Also presented are experimental results and empirical formulations obtained from the literature.

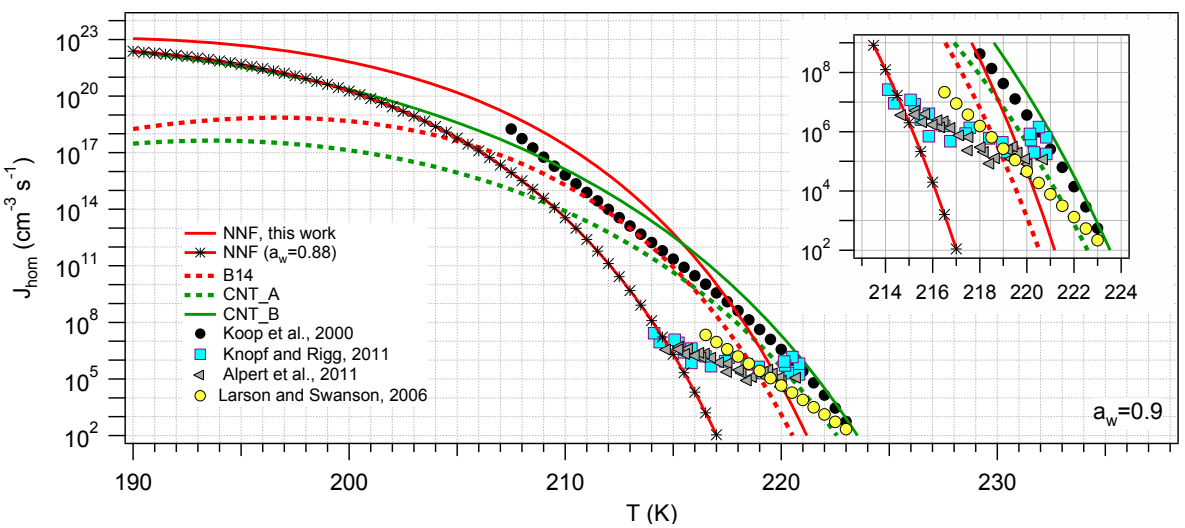

Figure 5. Homogeneous ice nucleation rate calculated using Eq. (16) (labeled "CNT") and Eq. (11) (labeled "NNF"). For CNT_A $\Delta G_{\text {act }}$ was defined as in Zobrist et al. (2007), and for CNT_B it was calculated using Eq. (14); in both cases the empirical expression for $\sigma_{\text {iw }}$ from Barahona (2014) was used. For the model of Koop et al. (2000), $a_{\mathrm{w}, \mathrm{eq}}$ was defined as in Koop and Zobrist (2009). Also presented are experimental results and empirical formulations obtained from the literature.

the activation energy. The correlation derived by Koop et al. (2000) is also reproduced along with available experimental data (Alpert et al., 2011; Knopf and Rigg, 2011). For the latter, only data reported for $T<221 \mathrm{~K}$ are shown to avoid heterogeneous freezing effects. The expression for $J_{\text {hom }}$ in $\left(\mathrm{NH}_{4}\right)_{2} \mathrm{SO}_{4}$ solutions derived by Larson and Swanson (2006) is also presented in Fig. 5. For the latter, the solution equilibrium model of Clegg et al. (1998) was used to relate $a_{\mathrm{w}}$ to the mass fraction of $\left(\mathrm{NH}_{4}\right)_{2} \mathrm{SO}_{4}$.

For $T>218 \mathrm{~K}$ and $a_{\mathrm{w}}=0.9, J_{\mathrm{hom}}$ from all formulations agree within 3 orders of magnitude and within experimental uncertainty (Alpert et al., 2011; Larson and Swanson, 2006) of the measured rates. For $T<216 \mathrm{~K}$, the models tend to predict higher than measured nucleation rates. Experimental measurements also show a weaker dependency of $J_{\text {hom }}$ on $T$ at $a_{\mathrm{w}}=0.9$ than at $a_{\mathrm{w}}=1.0$. This is evident in the data of Alpert et al. (2011) and less evident for the correlation of Larson and Swanson (2006). For the latter the authors report a $T$ uncertainty of about $1 \mathrm{~K}$, which may account for the observed difference. Alpert et al. (2011) reports an uncertainty in initial $a_{\mathrm{w}}$ of 0.01 , which explains the scatter of the data around $T \sim 218 \mathrm{~K}$. The authors, however, do not report measurements of $a_{\mathrm{w}}$ at the point of freezing. Knopf and Rigg (2011), who used a similar technique, discuss the possibility of a slight decrease in $a_{\mathrm{w}}$ of their test solutions as $T$ decreases, which would indicate a higher uncertainty in $a_{\mathrm{w}}$ than reported by Alpert et al. (2011). Similarly, Larson and Swanson (2006) performed their experiments at constant mass fraction and variation in $a_{\mathrm{w}}$ cannot be ruled out. To demonstrate the effect of a drift towards lower $a_{\mathrm{w}}$ values, $J_{\text {hom }}$ was recalculated at $a_{\mathrm{w}}=0.88$ (Fig. 5). It was found that a decrease of 0.02 in $a_{\mathrm{w}}$ with respect to the initial setting 
(assumed to be $a_{\mathrm{w}}=0.9$ ) would explain the observed $J_{\text {hom }}$. It is, however, remarkable that two different experimental techniques (Larson and Swanson, 2006; Alpert et al., 2011) show results consistent with a lower sensitivity of $J_{\text {hom }}$ to $T$ than predicted by theoretical models, indicating that such a discrepancy may not result solely from experimental artifacts. More research and further experimentation is required to clarify this point.

At a low temperature $(T<210 \mathrm{~K})$, the usage of Eq. (14) leads to a higher $J_{\text {hom }}$ than when $\Delta G_{\text {act, } 07}$ is used for both formulations of CNT. For $a_{\mathrm{w}}<1$ Eqs. (16) and (11) do not overlap as is the case for $a_{\mathrm{w}}=1$, which results from the different sensitivity to $a_{\mathrm{w}}$ of both formulations (Fig. 5). Interestingly, for $a_{\mathrm{w}}=1$ and $a_{\mathrm{w}}=0.9, J_{\mathrm{hom}}$ reaches similar values at $T=180 \mathrm{~K}$, being just about 1 order of magnitude higher in the latter due to the sensitivity of $\Delta G_{\text {act }}$ to $a_{\mathrm{w}}$. This shows that at a low $T, J_{\text {hom }}$ is mainly controlled by $J_{0}$ and hence by $\Delta G_{\text {act }}$.

Finally it is important to discuss the effect of variation in the parameters of the proposed theory on $J_{\text {hom }}$. It was concluded in B14 (cf., Fig. 8) that the uncertainty in $J_{\text {hom }}$ from variation in $\Gamma_{\mathrm{w}}$ and $s$ (used in Eq. 3 ) is about 2 orders of magnitude and decreases with decreasing $T$ since $J_{\text {hom }}$ becomes more dependent on $\Delta G_{\text {act }}$ and less dependent on the interfacial energy. Uncertainty in $\Delta G_{\text {act }}$ results mostly from uncertainty in $a_{\mathrm{w}, \mathrm{eq}}$ and $n_{t}$. The specification of the thermodynamic properties of water at very low $T$ is challenging since several combinations of parameters can lead to thermodynamically consistent solutions. Several studies (e.g., Johari et al., 1994; Koop and Zobrist, 2009) have used some form of thermodynamic continuation below $T \sim 235 \mathrm{~K}$ to define $a_{\mathrm{w}, \text { eq }}$ and $\Delta h_{\mathrm{f}}$, which is also used in this work. This approximation is indirectly supported by experimental results (Koop and Zobrist, 2009; Murphy and Koop, 2005; Johari et al., 1994); however, it may lead to uncertainty. It is estimated that the preexponential factor would increase by about 2 orders of magnitude with a change in $n_{t}$ from 16 to 15 . Essentially, a value of $n_{t}$ less than 16 would indicate that some of the rearrangement routes to form the transient state are prohibited. Another assumption that may impact the model is that of microscopic reversibility used in the derivation of $f\left(T, a_{\mathrm{w}}\right)$ (Sect. 2$)$, which becomes weak at very low $T$ since water dynamics becomes slow and it cannot be always assumed that the water molecules are in equilibrium with their local environment. Unfortunately, it is challenging to estimate a plausible range of uncertainty in $J_{\text {hom }}$ associated with these approximations. Deviations from thermodynamic equilibrium are difficult to quantify, even with molecular dynamics methods, and the characteristics of the transient state are not known. Progress in MD and further experimentation may shed light on these issues.

\section{Conclusions}

This work advances a theoretical description of the process of the interface transfer of water molecules from the liquid phase to ice during the early stages of nucleation. Unlike previous approaches, the model presented here does not assume that the water properties in the liquid-ice interface are the same as those of the bulk. Instead a theoretical approach is proposed where the interaction of several water molecules is required for interface transfer. The application of this model resulted in a thermodynamic definition of $\Delta G_{\text {act }}$. As $D_{\infty}$ and $\sigma_{\text {iw }}$ can also be defined on a thermodynamic basis (Adam and Gibbs, 1965; Barahona, 2014), this work gives support to the assertion of Koop et al. (2000) that the ice nucleation rate can be determined entirely by thermodynamics.

The approach proposed here elucidates two contributions to the activation energy. The first one is the self-diffusion process in the bulk water, that is, the breaking of hydrogen bonds in the liquid phase. The second is the work dissipated during interface transfer, associated with the rearrangement of the water molecules within the ice-liquid interface. The commonly used model of Turnbull and Fisher (1949) neglects the latter. However, since homogeneous ice nucleation occurs away from equilibrium, interface transfer implies an energy cost to the system. At temperatures relevant for homogeneous ice nucleation it represents about half of $\Delta G_{\text {act }}$.

It was shown that at a low temperature, interface transfer has the largest effect on the nucleation rate. For such conditions $\Delta G_{\text {act }} \sim \Delta G_{\text {hom }}$ and variations in the preexponential factor may dominate the variation in $J_{\text {hom }}$. On the other hand, moderate variation in $\Delta G_{\text {act }}$ will have a limited effect on $J_{\text {hom }}$ for pure water droplets since they typically freeze at $T>230 \mathrm{~K}$ where $\Delta G_{\text {hom }} \gg \Delta G_{\text {act }}$. However, $\Delta G_{\text {act }}$ may have a marked influence on the homogeneous freezing of haze aerosol, which occurs at a very low temperature. Also $\Delta G_{\text {act }}$ may impact the nucleation rate when the same formulation is used for heterogeneous ice nucleation as the nucleation work is typically lower than in the homogeneous case.

For $T>230 \mathrm{~K}$ the formulation of $\Delta G_{\text {act }}$ presented here predicts values close to those obtained using empirical formulations, particularly that of Zobrist et al. (2007). However, for $T<230 \mathrm{~K}$, Eq. (14) predicts a linear increase in $\Delta G_{\text {act }}$ with decreasing $T$ and differs from the nonlinear tendency typically found when $\Delta G_{\text {act }}$ is assumed to be determined solely by the self-diffusivity of bulk water (Ickes et al., 2015). As a result, at low $T$ the preexponential factor, and hence the nucleation rate, predicted using empirical formulations of $\Delta G_{\text {act }}$, tends to be lower than found in this work.

Introducing the new formulation of $\Delta G_{\text {act }}$ into a generalized form of CNT (Eq. 1) and using the NNF framework to define $\Delta G_{\text {hom }}$ resulted in good agreement of $J_{\text {hom }}$ with observations, even at very low $T$ where it is underestimated by most models. This is remarkable since no parameters of the theory were found by fitting nucleation rates. Introducing Eq. (14) into a common formulation of CNT with $\sigma_{\text {iw }}$ 
constrained as in B14 also led to a good agreement of $J_{\text {hom }}$ with measured values. For $a_{\mathrm{w}}=0.9$ and $T>218 \mathrm{~K}$, predicted $J_{\text {hom }}$ is in agreement within experimental uncertainty with reported experimental values; however, it tends to be higher than measurements at lower $T$. It is not clear whether systematic deviation in $a_{\mathrm{w}}$ during the experiments, or unknown factors not considered in the theoretical models are the source of this discrepancy, and more research is needed to elucidate this point. The NNF model, which can be independently constrained and evaluated, may be more suitable to investigate such differences between theory and measurements than common formulations of CNT where $\Delta G_{\text {act }}$ and $\sigma_{\text {iw }}$ must be fitted to measured $J_{\text {hom }}$.

This work centers on the activation energy as a fundamental parameter. Equation (13), however, suggests that the flux of water molecules from the bulk to the ice may be better understood in terms of the bulk self-diffusivity of water and the probability of interface transfer, $f\left(T, a_{\mathrm{w}}\right)$. These two quantities have a more specific physical meaning than $\Delta G_{\text {act }}$. $D_{\infty}$ has been independently measured (e.g., Smith and Kay, 1999), whereas $f\left(T, a_{\mathrm{w}}\right)$ is related to the work dissipated during ice nucleation and can in principle be obtained from MD simulations.

More work is needed to estimate the range of validity of the approximations introduced in Sect. 2. Guided by MD results, it was assumed that a molecule crossing the interface would interact with four other molecules, so that $n_{\mathrm{t}}=16$. This is expected at low $T$ since the water structure becomes more ice-like; however, $n_{\mathrm{t}}$ may be a function of the temperature. For example, the size of cooperative regions in water is known to be a function of the configurational entropy and therefore of temperature (Adam and Gibbs, 1965). It is not clear whether that should also be the case for interface transfer. The role of surface-stimulated nucleation (Djikaev, 2008) and the effect of deviations from microscopic reversibility on $\Delta G_{\text {act }}$ and $J_{\text {hom }}$ must be further investigated. The estimation of water properties at low $T$ is also challenging and still uncertain. Progress in MD and further experimentation are required to address these issues. Understanding $\Delta G_{\text {act }}$ as a thermodynamic function, instead of a fundamental property of water, may help set up a road map for further research.

From their analysis of different models, Ickes et al. (2015) concluded that at low $T$ either $\sigma_{\text {iw }}$ is thermodynamically undefined or the temperature dependency of $\Delta G_{\text {act }}$ reverses. This work shows that both $\Delta G_{\text {act }}$ and $\sigma_{\text {iw }}$ can be defined on a thermodynamic basis. The work of Ickes et al. (2015), however, shows the difficulties in ascribing physical behavior to the parameters of CNT by fitting experimental results. The independent theoretical formulation presented here may be more amenable to testing and expansion. In turn, a physically based definition of the parameters of CNT may improve the development of parameterizations of ice formation in cloud models and lead to a better understanding of ice processes in the atmosphere.
Acknowledgements. Donifan Barahona was supported by the NASA Modeling, Analysis and Prediction program under WBS 802678.02.17.01.25.

Edited by: D. Knopf

\section{References}

Adam, G. and Gibbs, J. H.: On the temperature dependence of cooperative relaxation properties in glass-forming liquids, J. Chem. Phys., 43, 139-146, 1965.

Alpert, P. A., Aller, J. Y., and Knopf, D. A.: Ice nucleation from aqueous $\mathrm{NaCl}$ droplets with and without marine diatoms, Atmos. Chem. Phys., 11, 5539-5555, doi:10.5194/acp-11-55392011, 2011.

Barahona, D.: Analysis of the effect of water activity on ice formation using a new thermodynamic framework, Atmos. Chem. Phys., 14, 7665-7680, doi:10.5194/acp-14-7665-2014, 2014

Barahona, D. and Nenes, A.: Parameterization of cirrus formation in large scale models: Homogeneous nucleation, J. Geophys. Res., 113, D11211, doi:10.1029/2007JD009355, 2008.

Barahona, D. and Nenes, A.: Dynamical states of low temperature cirrus, Atmos. Chem. Phys., 11, 3757-3771, doi:10.5194/acp11-3757-2011, 2011.

Barahona, D., Rodriguez, J., and Nenes, A.: Sensitivity of the global distribution of cirrus ice crystal concentration to heterogeneous freezing, J. Geophys. Res., 15, D23213, doi:10.1029/2010JD014273, 2010.

Barahona, D., Molod, A., Bacmeister, J., Nenes, A., Gettelman, A., Morrison, H., Phillips, V., and Eichmann, A.: Development of two-moment cloud microphysics for liquid and ice within the NASA Goddard Earth Observing System Model (GEOS-5), Geosci. Model Dev., 7, 1733-1766, doi:10.5194/gmd-7-17332014, 2014.

Bartell, S. L. and Chushak, Y. G.: Nucleation of Ice in Large Water Clusters: Experiment and Simulation, in: Water in Confining Geometries, edited by: Buch, V. and Devlin, J. P., Springer Science and Business Media, New York, p. 399-424, 2003.

Buhariwalla, C. R., Bowles, R. K., Saika-Voivod, I., Sciortino, F., and Poole, P. H.: Free energy of formation of small ice nuclei near the Widom line in simulations of supercooled water, arXiv preprint arXiv:1501.03115, 2015.

Bullock, G. and Molinero, V.: Low-density liquid water is the mother of ice: on the relation between mesostructure, thermodynamics and ice crystallization in solutions, Faraday Discuss., 167, 371-388, doi:10.1039/C3FD00085K, 2013.

Bustamante, C., Liphardt, J., and Ritort, F.: The nonequilibrium thermodynamics of small systems, Phys. Today, 58, 43-4, doi:10.1063/1.2012462, 2005.

Clegg, S. L., Brimblecombe, P., and Wexler, A. S.: Thermodynamic model of the system $\mathrm{H}+-\mathrm{NH} 4+-\mathrm{SO} 42-\mathrm{NO} 3-\mathrm{H} 2 \mathrm{O}$ at tropospheric temperatures, J. Phys. Chem. A, 102, 2137-2154, 1998.

Crooks, G. E.: Entropy production fluctuation theorem and the nonequilibrium work relation for free energy differences, Physical Review E, 60, 2721, doi:10.1103/PhysRevE.60.2721, 1999.

Digilov, R. M.: Semi-empirical model for prediction of crystal-melt interfacial tension, Surface Sci., 555, 68-74, 2004. 
Djikaev, Y. S.: Effect of the surface-stimulated mode on the kinetics of homogeneous crystal nucleation in droplets, J. Phys. Chem. A, 112, 6592-6600, 2008.

Einstein, A.: Investigation on the theory of the Brownian movement, Dover Publications Inc., New York, USA, 1956.

Espinosa, J., Sanz, E., Valeriani, C., and Vega, C.: Homogeneous ice nucleation evaluated for several water models, J. Chem. Phys., 141, 18C529, doi:10.1063/1.4897524, 2014.

Espinosa, J., Sanz, E., Valeriani, C., and Vega, C.: Homogeneous ice nucleation evaluated for several water models, J. Chem. Phys., 141, 18C529, doi:10.1063/1.4897524, 2014.

Hagen, D. E., Anderson, R. J., and Kassner Jr, J. L.: Homogeneous condensation-freezing nucleation rate measurements for small water droplets in an expansion cloud chamber, J. Atmos. Sci., 38, 1236-1243, 1981.

Ickes, L., Welti, A., Hoose, C., and Lohmann, U.: Classical nucleation theory of homogeneous freezing of water: thermodynamic and kinetic parameters, Phys. Chem. Chem. Phys., 17, 55145537, doi:10.1039/C4CP04184D, 2015.

Jeffery, C. and Austin, P.: Homogeneous nucleation of supercooled water: Results from a new equation of state, J. Geophys. Res., 102, 25269-25279, 1997.

Jensen, E. J., Diskin, G., Lawson, R. P., Lance, S., Bui, T. P., Hlavka, D., McGill, M., Pfister, L., Toon, O. B., and Gao, R.: Ice nucleation and dehydration in the Tropical Tropopause Layer, P. Natl. Acad. Sci. USA, 110, 2041-2046, 2013.

Johari, G., Fleissner, G., Hallbrucker, A., and Mayer, E.: Thermodynamic continuity between glassy and normal water, J. Phys. Chem., 98, 4719-4725, 1994.

Jou, D., Casas-Vázquez, J., and Lebon, G.: Extended Irreversible Thermodynamics, 4th edn., Springer, the Netherlands, 2010.

Kärcher, B. and Burkhardt, U.: A cirrus scheme for global circulation models, Q. J. Roy. Meteorol. Soc., 134, 1439-1461, doi:10.1002/Qj.301, 2008.

Kärcher, B. and Lohmann, U.: A parameterization of cirrus cloud formation: homogeneous freezing including effects or aerosol size, J. Geophys. Res., 107, 4698, doi:10.1029/2001JD001429, 2002.

Kashchiev, D.: Nucleation: Basic Theory with Applications, Butterworth-Heinemann, Oxford, 2000.

Kawasaki, T. and Tanaka, H.: Formation of a crystal nucleus from liquid, P. Natl. Acad. Sci. USA, 107, 14036-14041, 2010.

Kay, J. E., Tsemekhman, V., Larson, B., Baker, M., and Swanson, B.: Comment on evidence for surface-initiated homogeneous nucleation, Atmos. Chem. Phys., 3, 1439-1443, doi:10.5194/acp3-1439-2003, 2003.

Khvorostyanov, V. I. and Curry, J. A.: Thermodynamic theory of freezing and melting of water and aqueous solutions, J. Phys. Chem. A, 108, 11073-11085, 2004.

Khvorostyanov, V. and Curry, J.: Critical humidities of homogeneous and heterogeneous ice nucleation: Inferences from extended classical nucleation theory, J. Geophys. Res., 114, D04207, doi:10.1029/2008JD011197, 2009.

Knopf, D. A. and Rigg, Y. J.: Homogeneous ice nucleation from aqueous inorganic/organic particles representative of biomass burning: Water activity, freezing temperatures, nucleation rates, J. Phys. Chem. A, 115, 762-773, 2011.
Koop, T. and Zobrist, B.: Parameterizations for ice nucleation in biological and atmospheric systems, Phys. Chem. Chem. Phys., 11, 10839-10850, 2009.

Koop, T., Luo, B., Tslas, A., and Peter, T.: Water activity as the determinant for homogeneous ice nucleation in aqueous solutions, Nature, 406, 611-614, 2000.

Larson, B. H. and Swanson, B. D.: Experimental investigation of the homogeneous freezing of aqueous ammonium sulfate droplets, J. Phys. Chem. A, 110, 1907-1916, 2006.

Liu, X. and Penner, J.: Ice nucleation parameterization for global models, Meteorol. Z., 14, 499-514, 2005.

Lohmann, U. and Kärcher, B.: First interactive simulations of cirrus clouds formed by homogeneous freezing in the ECHAM general circulation model, J. Geophys. Res., 107, 4105, doi:10.1029/2001JD000767, 2002.

Malkin, T. L., Murray, B. J., Brukhno, A. V., Anwar, J., and Salzmann, C. G.: Structure of ice crystallized from supercooled water, P. Natl. Acad. Sci. USA, 109, 1041-1045, 2012.

Manka, A., Pathak, H., Tanimura, S., Wölk, J., Strey, R., and Wyslouzil, B. E.: Freezing water in no-man's land, Phys. Chem. Chem. Phys., 14, 4505-4516, 2012.

Matsumoto, M., Saito, S., and Ohmine, I.: Molecular dynamics simulation of the ice nucleation and growth process leading to water freezing, Nature, 416, 409-413, 2002.

Moore, E. B. and Molinero, V.: Structural transformation in supercooled water controls the crystallization rate of ice, Nature, 479 , 506-508, 2011.

Moroni, D., Ten Wolde, P. R., and Bolhuis, P. G.: Interplay between structure and size in a critical crystal nucleus, Phys. Rev. Lett., 94, 235703, doi:10.1103/PhysRevLett.94.235703, 2005.

Murphy, D. and Koop, T.: Review of the vapour pressures of ice and supercooled water for atmospheric applications, Q. J. Roy. Meteorol. Soc., 131, 1539-1565, 2005.

Murray, B., Broadley, S., Wilson, T., Bull, S., Wills, R., Christenson, H., and Murray, E.: Kinetics of the homogeneous freezing of water, Phys. Chem. Chem. Phys., 12, 10380-10387, 2010.

Pruppacher, H.: A new look at homogeneous ice nucleation in supercooled water drops, J. Atmos. Sci., 52, 1924-1933, 1995.

Pruppacher, H. and Klett, J.: Microphysics of clouds and precipitation, Kluwer Academic Publishers, Boston, MA, 2nd edn., 1997.

Reinhardt, A. and Doye, J. P.: Note: homogeneous TIP4P/2005 ice nucleation at low supercooling, J. Chem. Phys., 139, 096102, doi:10.1063/1.4819898, 2013.

Riechers, B., Wittbracht, F., Hütten, A., and Koop, T.: The homogeneous ice nucleation rate of water droplets produced in a microfluidic device and the role of temperature uncertainty, Phys. Chem. Chem. Phys., 15, 5873-5887, 2013.

Røsjorde, A., Fossmo, D., Bedeaux, D., Kjelstrup, S., and Hafskjold, B.: Nonequilibrium molecular dynamics simulations of steady-state heat and mass transport in condensation: I. Local equilibrium, J. Colloid Interface Sci., 232, 178-185, 2000.

Russo, J., Romano, F., and Tanaka, H.: New metastable form of ice and its role in the homogeneous crystallization of water, Nature Materials, 13, 733-739, 2014.

Sigurbjörnsson, Ó. F. and Signorell, R.: Volume versus surface nucleation in freezing aerosols, Phys. Rev. E, 77, 051601, doi:10.1103/PhysRevE.77.051601, 2008. 
Singh, R. S. and Bagchi, B.: Correlation between thermodynamic anomalies and pathways of ice nucleation in supercooled water, J. Chem. Phys., 140, 164503, doi:10.1063/1.4871388, 2014.

Smith, R. S. and Kay, B. D.: The existence of supercooled liquid water at $150 \mathrm{~K}$, Nature, 398, 788-791, 1999.

Spaepen, F.: A structural model for the solid-liquid interface in monatomic systems, Acta Metall. Mater., 23, 729-743, 1975.
Taborek, P.: Nucleation in emulsified supercooled water, Phys. Rev. B, 32, 5902, 1985.

Turnbull, D. and Fisher, J. C.: Rate of nucleation in condensed systems, J. Chem. Phys., 17, 71-73, 1949.

Zobrist, B., Koop, T., Luo, B., Marcolli, C., and Peter, T.: Heterogeneous ice nucleation rate coefficient of water droplets coated by a nonadecanol monolayer, J. Phys. Chem. C, 111, 2149-2155, 2007. 